Revised on July 14, 2001

\title{
Hyperbolicity of Partition Function and Quantum Gravity
}

\author{
Kazuhiro HikAmi \\ Department of Physics, Graduate School of Science, \\ University of Tokyo, \\ Hongo 7-3-1, Bunkyo, Tokyo 113-0033, Japan.
}

(Received: $\quad$ )

\begin{abstract}
We study a geometry of the partition function which is defined in terms of a solution of the five-term relation. It is shown that the 3-dimensional hyperbolic structure or Euclidean $\mathrm{AdS}_{3}$ naturally arises in the classical limit of this invariant. We discuss that the oriented ideal tetrahedron can be assigned to the partition function of string.
\end{abstract}

PACS: 04.60.-m, 04.60.Nc, 03.65.-w

\footnotetext{
$\dagger$ hikami@phys.s.u-tokyo.ac.jp
} 


\section{Introduction}

This article is devoted to reveal a relationship between the partition function and 3dimensional hyperbolic geometry. From the physical viewpoint, the 3-dimensional hyperbolic space $\mathbb{H}^{3}$ corresponds to the Euclidean Anti-de Sitter space $\mathrm{AdS}_{3}$, and many studies concerning the AdS/CFT correspondence [1] including string theory on AdS and the $S L(2, \mathbb{R})$ WZNW model have been done (for a review, see Ref. 2 and references therein). Our motivation is based on a recent conjecture that an asymptotic behavior of a specific value of the colored Jones polynomial gives the hyperbolic volume of knot complement [3]. This opens up a geometrical study of the quantum knot invariants such as the Jones polynomial, which have been introduced based on the quantum group. Here

we shall give a geometrical picture of the partition function which indicates an aspect of the AdS/CFT correspondence; we show that the bosonic and fermionic partition function constitutes as oriented ideal tetrahedron in $\mathbb{H}^{3}$. This partition function can be seen as a string partition function on knot, which is a collection of torus and is on the boundary of $\mathbb{H}^{3}$.

The organization of this paper is as follows. In section 2 we briefly review a fact of the hyperbolic space $\mathbb{H}^{3}$. Almost all orientable 3-manifolds are believed to admit a hyperbolic structure, and the hyperbolic volume of manifold as invariant can be constructed based on the ideal triangulation. In section 3 we study several properties of the quantum dilogarithm function. Key identity is the five-term relation, and we define the partition function by assigning oriented tetrahedron to the quantum dilogarithm function. In section 1 we study a classical limit of the partition function. We show that the oriented tetrahedron can be regarded as the ideal tetrahedron in $\mathbb{H}^{3}$, and that the hyperbolicity consistency condition in gluing tetrahedra exactly coincides with the critical point of the partition function. In section 5 we consider an application in physics, and we clarify a relationship with the AdS/CFT correspondence. As was pointed out in Refs. 9, 10, the quantum dilogarithm function used here is a modular double of the $q$-exponential function, which coincides with the partition function of string from cylinder. We show that the chemical potential and the 
average number of angular momentum determines the ideal tetrahedron. The last section is for concluding remarks.

\section{Hyperbolic Space $\mathbb{H}^{3}$}

The 3-dimensional hyperbolic space $\mathbb{H}^{3}$ (see e.g. Refs. 11, 12), or the Euclidean version of Anti-de Sitter space $\mathrm{AdS}_{3}$, is defined as the space-like surface in 4-dimensional Minkowski space;

$$
-x_{0}^{2}+x_{1}^{2}+x_{2}^{2}+x_{3}^{2}=-1, \quad x_{0}>0
$$

Here the metric is $\mathrm{d} s^{2}=-\mathrm{d} x_{0}{ }^{2}+\mathrm{d} x_{1}{ }^{2}+\mathrm{d} x_{2}{ }^{2}+\mathrm{d} x_{3}{ }^{2}$, and it has a constant negative curvature -1 . The hyperbolic space $\mathbb{H}^{3}$ is conformally mapped into the 3-dimensionally disk $\mathbb{D}_{3}$ (the Poincaré model) via

$$
\begin{gathered}
x \mapsto \frac{\left(x_{1}, x_{2}, x_{3}\right)}{1+x_{0}}, \\
\begin{cases}x_{0}=\operatorname{ch} \xi, & x_{1}=\operatorname{sh} \xi \sin \theta \cos \varphi \\
x_{2}=\operatorname{sh} \xi \sin \theta \sin \varphi, & x_{3}=\operatorname{sh} \xi \cos \theta\end{cases}
\end{gathered}
$$

with $\mathrm{d} s^{2}=\mathrm{d} \xi^{2}+\operatorname{sh}^{2} \xi\left(\mathrm{d} \theta^{2}+\sin ^{2} \theta \mathrm{d} \varphi^{2}\right)$, and boundary $\partial \mathbb{H}^{3}$ of the hyperbolic space is homeomorphic to $S^{2}$. The orientation-preserving isometry of $\mathbb{H}^{3}$ is isomorphic to $P S L(2, \mathbb{C})$. Hereafter to consider the ideal tetrahedron, we use the Beltrami half-space model $X_{0}>0$ with coordinate $\left(X_{0}, X_{1}, X_{2}\right)$, and the metric is given by

$$
\mathrm{d} s^{2}=\frac{\mathrm{d} X_{0}{ }^{2}+\mathrm{d} X_{1}{ }^{2}+\mathrm{d} X_{2}{ }^{2}}{X_{0}{ }^{2}} .
$$

The transformation is explicitly given by

$$
X_{0}^{-1}=\operatorname{ch} \xi+\operatorname{sh} \xi \cos \theta, \quad X_{1}=X_{0} \operatorname{sh} \xi \sin \theta \cos \varphi, \quad X_{2}=X_{0} \operatorname{sh} \xi \sin \theta \sin \varphi .
$$

With this metric geodesics are semi-circles orthogonal to $\left\{X_{0}=0\right\} \cup \infty$.

Every non-compact finite volume hyperbolic 3-manifold admits a decomposition into a finite number of ideal polyhedra [11, 13]. The ideal polyhedron means that all vertices 
lie on $\partial \mathbb{H}^{3}$, and all of edges are hyperbolic geodesics. Among them ideal tetrahedron is completely determined by a single complex number $z$ with positive imaginary part, which we call modulus, or cross-ratio; this follows from that the ideal tetrahedron is determined by the three dihedral angels $\alpha, \beta$, and $\gamma$ of edges satisfying $\alpha+\beta+\gamma=\pi$. For fixed edge $e$, the parameter which describes the dihedral angle (see Fig. 1) is given in terms of modulus by

$$
z_{i}(e) \in\left\{z_{i}, \frac{1}{1-z_{i}}, 1-\frac{1}{z_{i}}\right\} .
$$

The hyperbolic volume of the ideal tetrahedron is given by the Bloch-Wigner function $D(z)$ (see eq. (A.3) 14. Depending on the orientation of tetrahedron, we can extend the modulus to $z \in \mathbb{C}$.

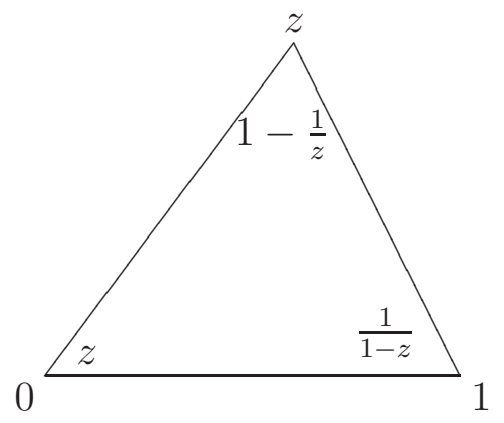

Figure 1:

Gluing a finite collection of ideal tetrahedra together results in a 3-manifold $M$ admitting a hyperbolic structure of finite volume [11]. To endow the hyperbolic structure in $M$, the following gluing conditions must be satisfied;

1. triangles cut out of adjacent tetrahedra to every edge $e_{\nu}$ to fill neatly around edge, i.e., we require the consistency condition

$$
\sum_{j} \arg z_{j}\left(e_{\nu}\right)=2 \pi, \quad \quad \prod_{j} z_{j}\left(e_{\nu}\right)=1 .
$$

2. the developing map near the ideal vertex yields a Euclidean structure on the horosphere. 
i.e. an ideal triangulation of a 3-manifold $M$, when $\partial M$ is a collection of tori, $M-\partial M$ have a hyperbolic structure if and only if for each edge the hyperbolic consistency condition is fulfilled. It is not clear for a case of closed manifold, which can be obtained topologically by Dehn filling a certain hyperbolic link complement. When a 3-manifold $M$ is triangulated in this way, a sum of the hyperbolic volume of the ideal tetrahedron

$$
\operatorname{Vol}(M)=\sum_{i} D\left(z_{i}\right)
$$

is a topological invariant of $M$.

The volume of oriented hyperbolic 3-manifold $M$ has an analytic relationship with the Chern-Simons invariant CS $(M)$ [15, 16]. Namely

$$
\operatorname{VCS}(M)=\operatorname{Vol}(M)+\mathrm{i} C S(M)
$$

is a natural complexification of $\operatorname{Vol}(M)$, and in general the formulae to compute $\operatorname{CS}(M)$ give $\operatorname{Vol}(M)$. The invariant $\operatorname{VCS}(M)$ is induced from the pre-Bloch group [17] by

$$
\begin{aligned}
\mathrm{i} \operatorname{VCS}: \mathcal{P}(\mathbb{C}) & \longrightarrow \mathbb{C} \\
{\left[z_{i}\right] } & \longmapsto L\left(1-z_{i}\right)
\end{aligned}
$$

Here $[z]$ is the element of the Bloch group satisfying

$$
[x]-[y]+\left[\frac{y}{x}\right]-\left[\frac{1-x^{-1}}{1-y^{-1}}\right]+\left[\frac{1-x}{1-y}\right]=0,
$$

and $L(z)$ is the Rogers dilogarithm function (A.2). Fact that eqs. (2.1) and (2.2) are invariant of $M$ is based on that the Bloch invariant defined by

$$
\beta(M)=\sum_{i}\left[z_{i}\right]
$$

is the invariant of the manifold [18].

\section{Quantum Dilogarithm Function and Partition Function}

We study the integral,

$$
\Phi_{\gamma}(\varphi)=\exp \left(\int_{\mathbb{R}+\mathrm{i} 0} \frac{\mathrm{e}^{-\mathrm{i} \varphi x}}{4 \operatorname{sh}(\gamma x) \operatorname{sh}(\pi x)} \frac{\mathrm{d} x}{x}\right),
$$


which was first introduced by Faddeev [9]. As we will discuss later, this function was originally introduced as a dualization or modular double of the $q$-exponential function. The function enjoys the five-term relation [19, 20],

$$
\Phi_{\gamma}(\hat{p}) \Phi_{\gamma}(\hat{q})=\Phi_{\gamma}(\hat{q}) \Phi_{\gamma}(\hat{p}+\hat{q}) \Phi_{\gamma}(\hat{p})
$$

where $\hat{p}$ and $\hat{q}$ are the canonically conjugate operators satisfying

$$
[\hat{p}, \hat{q}]=-2 \mathrm{i} \gamma
$$

The five-term relation (3.2) is rewritten into simple form,

$$
S_{2,3} S_{1,2}=S_{1,2} S_{1,3} S_{2,3}
$$

where the $S$-operator is defined by

$$
S_{1,2}=\mathrm{e}^{\frac{1}{2 \mathrm{i} \gamma} \hat{q}_{1} \hat{p}_{2}} \cdot \Phi_{\gamma}\left(\hat{p}_{1}+\hat{q}_{2}-\hat{p}_{2}\right)
$$

Here $\hat{p}_{1}=\hat{p} \otimes \mathbb{1}, \hat{q}_{2}=\mathbb{1} \otimes \hat{q}$, and so on. The matrix element of the $S$-operator over the momentum space, $\hat{p}|p\rangle=p|p\rangle$, is given by [7]

$$
\begin{gathered}
\left\langle p_{1}, p_{2}|S| p_{1}^{\prime}, p_{2}^{\prime}\right\rangle=\delta\left(p_{1}+p_{2}-p_{1}^{\prime}\right) \cdot \Phi_{\gamma}\left(p_{2}^{\prime}-p_{2}+\mathrm{i} \pi+\mathrm{i} \gamma\right) \cdot \mathrm{e}^{\frac{1}{2 \mathrm{i} \gamma}\left(-\frac{\pi^{2}+\gamma^{2}}{6}-\frac{\gamma \pi}{2}+p_{1}\left(p_{2}^{\prime}-p_{2}\right)\right)} \\
\left\langle p_{1}, p_{2}\left|S^{-1}\right| p_{1}^{\prime}, p_{2}^{\prime}\right\rangle=\delta\left(p_{1}-p_{1}^{\prime}-p_{2}^{\prime}\right) \cdot \frac{1}{\Phi_{\gamma}\left(p_{2}-p_{2}^{\prime}-\mathrm{i} \pi-\mathrm{i} \gamma\right)} \cdot \mathrm{e}^{\frac{1}{2 \mathrm{i} \gamma}\left(\frac{\pi^{2}+\gamma^{2}}{6}+\frac{\gamma \pi}{2}-p_{1}^{\prime}\left(p_{2}-p_{2}^{\prime}\right)\right)} .
\end{gathered}
$$

We can give the geometrical picture for this operator; a key is the five-term relation (3.4). We associate the (3-dimensional) oriented tetrahedron to the $S$-operators; each 2-simplex is assigned the momentum (Fig. 2). With this identification, we can glue two 2-simplex in pairs so that the orientation of edges match. For instance, the pentagon relation (3.4) can be depicted as Fig. 3.

When 3-manifold $M$ is decomposed into ideal tetrahedron, we define the partition function $\tau(M)$ by

$$
\tau(M)=\iint \mathrm{d} p \prod\left\langle p_{i}, p_{j}\left|S^{ \pm 1}\right| p_{k}, p_{l}\right\rangle
$$

In the following we study in detail the classical limit of the partition function $\tau(M)$. 

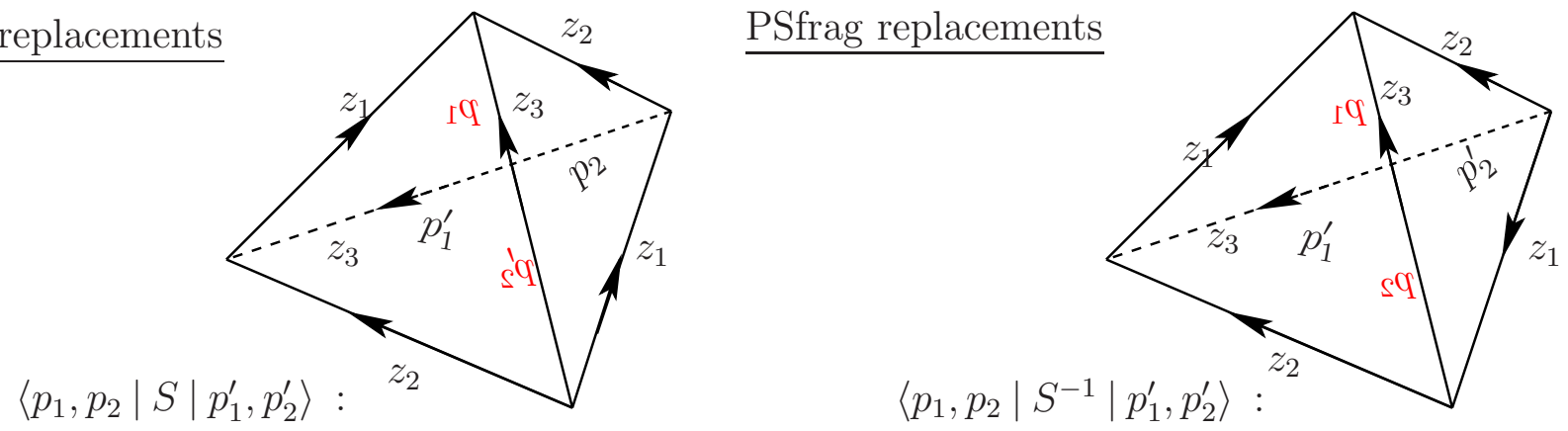

Figure 2: The $S$-operators (3.6) denote the oriented tetrahedron. Parameters $p$ are assigned to every face, and $z_{a}$ denotes a dihedral angle. In the classical limit $\gamma \rightarrow 0$, those become hyperbolic ideal tetrahedra with modulus $\mathrm{e}^{p_{2}^{\prime}-p_{2}}$, and the dihedral angles $z_{a}$ at the edges of the simplex are $z_{1}=\mathrm{e}^{p_{2}^{\prime}-p_{2}}, z_{2}=1-z_{1}{ }^{-1}$, and $z_{3}=\left(1-z_{1}\right)^{-1}$, respectively, with opposite edges having the same angle.
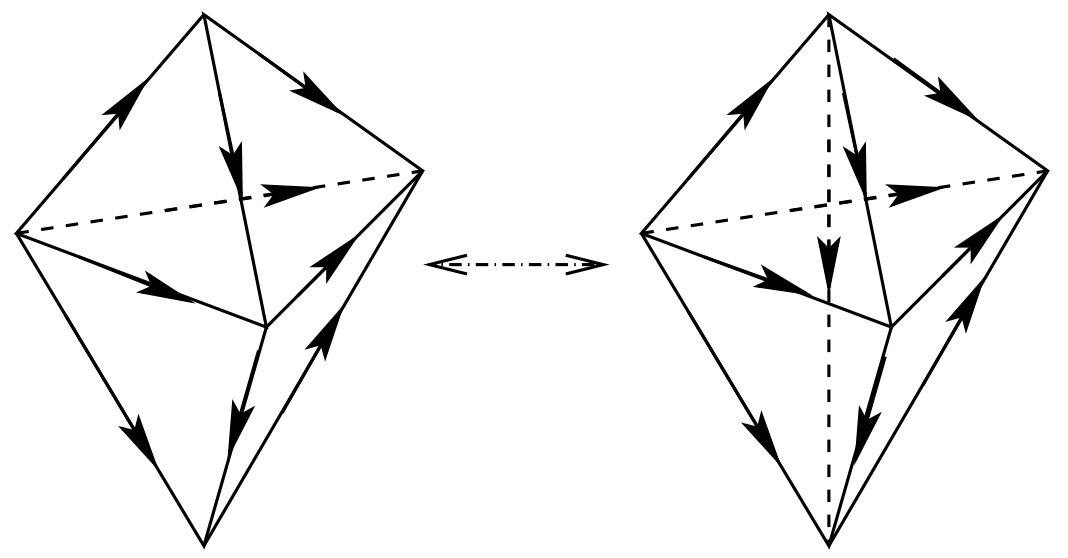

Figure 3: Pentagon identity (3.4). 


\section{Classical Limit and Hyperbolic Geometry}

We study the classical limit $\gamma \rightarrow 0$ of matrix elements (3.6) of the $S$-operators. As simple calculation leads that the Faddeev integral reduces to the Euler dilogarithm function (A.1)

$$
\Phi_{\gamma}(x)=\exp \frac{1}{2 \mathrm{i} \gamma}\left(\operatorname{Li}_{2}\left(-\mathrm{e}^{x}\right)+O(\gamma)\right)
$$

we have

$$
\begin{aligned}
& \left\langle p_{1}, p_{2}|S| p_{1}^{\prime}, p_{2}^{\prime}\right\rangle=\delta\left(p_{1}+p_{2}-p_{1}^{\prime}\right) \cdot \exp \left(-\frac{1}{2 \mathrm{i} \gamma} V\left(p_{2}^{\prime}-p_{2}, p_{1}\right)+O\left(\gamma^{0}\right)\right), \\
& \left\langle p_{1}, p_{2}\left|S^{-1}\right| p_{1}^{\prime}, p_{2}^{\prime}\right\rangle=\delta\left(p_{1}-p_{1}^{\prime}-p_{2}^{\prime}\right) \cdot \exp \left(\frac{1}{2 \mathrm{i} \gamma} V\left(p_{2}-p_{2}^{\prime}, p_{1}^{\prime}\right)+O\left(\gamma^{0}\right)\right),
\end{aligned}
$$

where

$$
V(x, y)=\frac{\pi^{2}}{6}-\mathrm{Li}_{2}\left(\mathrm{e}^{x}\right)-x y
$$

Thus the operators $S^{ \pm 1}$, which is assigned to oriented tetrahedron in the partition function (3.7), asymptotically reduces to the function $V(x, y)$ satisfying

$$
\begin{gathered}
V(x, y)=L\left(1-\mathrm{e}^{x}\right)+\frac{1}{2}\left(\frac{\partial V(x, y)}{\partial \log x}+\frac{\partial V(x, y)}{\partial \log y}\right) \\
\operatorname{Im} V(x, y)=D\left(1-\mathrm{e}^{x}\right)+\log \left|\mathrm{e}^{x}\right| \cdot \operatorname{Im}\left(\frac{\partial V(x, y)}{\partial x}\right)+\log \left|\mathrm{e}^{y}\right| \cdot \operatorname{Im}\left(\frac{\partial V(x, y)}{\partial y}\right) .
\end{gathered}
$$

Here we have used the Rogers dilogarithm (A.2) and the Bloch-Wigner function (A.3). As the Bloch-Wigner function $D(z)$ gives the hyperbolic volume of the ideal tetrahedron [14], we are forced to study a relationship between the $S$-operator at the critical point and the hyperbolic geometry.

For our purpose we reconsider the five-term relation (3.4) in the classical limit in detail. We substitute the asymptotic form (4.2) into matrix form of eq. (3.4), $\left\langle p_{1}, p_{2}, p_{3}\left|S_{2,3} S_{1,2}\right| p_{1}^{\prime}, p_{2}^{\prime}, p_{3}^{\prime}\right\rangle=\left\langle p_{1}, p_{2}, p_{3}\left|S_{1,2} S_{1,3} S_{2,3}\right| p_{1}^{\prime}, p_{2}^{\prime}, p_{3}^{\prime}\right\rangle$. LHS reduces to

$$
\begin{aligned}
& \int \mathrm{d} x\left\langle p_{2}, p_{3}|S| x, p_{3}^{\prime}\right\rangle\left\langle p_{1}, x|S| p_{1}^{\prime}, p_{2}^{\prime}\right\rangle \\
& =\delta\left(p_{1}+p_{2}+p_{3}-p_{1}^{\prime}\right) \cdot \exp \frac{1}{2 \mathrm{i} \gamma}\left(-V\left(p_{3}^{\prime}-p_{3}, p_{2}\right)-V\left(p_{2}^{\prime}-p_{2}-p_{3}, p_{1}\right)+O(\gamma)\right),
\end{aligned}
$$


while RHS gives

$$
\begin{gathered}
\iiint \mathrm{d} y \mathrm{~d} z \mathrm{~d} w\left\langle p_{1}, p_{2}|S| y, z\right\rangle\left\langle y, p_{3}|S| p_{1}^{\prime}, w\right\rangle\left\langle z, w|S| p_{2}^{\prime}, p_{3}^{\prime}\right\rangle \\
=\delta\left(p_{1}+p_{2}+p_{3}-p_{1}^{\prime}\right) \cdot \int \mathrm{d} z \exp \frac{1}{2 \mathrm{i} \gamma}\left(-\frac{\pi^{2}}{2}+\operatorname{Li}_{2}\left(\mathrm{e}^{z-p_{2}}\right)+\operatorname{Li}_{2}\left(\mathrm{e}^{p_{2}^{\prime}-p_{3}-z}\right)+\operatorname{Li}_{2}\left(\mathrm{e}^{p_{3}^{\prime}-p_{2}^{\prime}+z}\right)\right. \\
\left.+z\left(-p_{2}+p_{3}^{\prime}-p_{2}^{\prime}+z\right)-p_{1} p_{2}+\left(p_{2}^{\prime}-p_{3}\right)\left(p_{1}+p_{2}\right)+O(\gamma)\right) \cdot \quad(4.7)
\end{gathered}
$$

Above integral w.r.t. $z$ is evaluated by use of the saddle point method, whose critical point is given by

$$
\left(1-\mathrm{e}^{w-p_{3}}\right)^{-1} \cdot\left(1-\mathrm{e}^{p_{2}-z}\right) \cdot\left(1-\mathrm{e}^{w-p_{3}^{\prime}}\right)=1, \quad w=p_{2}^{\prime}-z
$$

In fact, substituting a solution

$$
\mathrm{e}^{-z}=\mathrm{e}^{-p_{2}}+\mathrm{e}^{-p_{2}^{\prime}+p_{3}^{\prime}}-\mathrm{e}^{-p_{2}-p_{3}+p_{3}^{\prime}}
$$

into the integral (4.7), and equating both hands sides, eq. (4.6) and eq. (4.7) at critical point, we get

$$
\begin{aligned}
\operatorname{Li}_{2}\left(\mathrm{e}^{z-p_{2}}\right)+\operatorname{Li}_{2}\left(\mathrm{e}^{p_{2}^{\prime}-p_{3}-z}\right)+\operatorname{Li}_{2}\left(\mathrm{e}^{p_{3}^{\prime}-p_{2}^{\prime}+z}\right)+\left(z-p_{2}\right)(z- & \left.p_{2}^{\prime}+p_{3}^{\prime}\right)-\frac{\pi^{2}}{6} \\
& =\operatorname{Li}_{2}\left(\mathrm{e}^{p_{3}^{\prime}-p_{3}}\right)+\operatorname{Li}_{2}\left(\mathrm{e}^{p_{2}^{\prime}-p_{2}-p_{3}}\right) .
\end{aligned}
$$

which is nothing but Schaeffer's five-term relation of the Euler dilogarithm function,

$$
\operatorname{Li}_{2}\left(\frac{1-x^{-1}}{1-y^{-1}}\right)=\operatorname{Li}_{2}(x)-\operatorname{Li}_{2}(y)+\operatorname{Li}_{2}\left(\frac{y}{x}\right)+\operatorname{Li}_{2}\left(\frac{1-x}{1-y}\right)-\frac{\pi^{2}}{6}+\log x \log \left(\frac{1-x}{1-y}\right),
$$

with $x=\mathrm{e}^{z-p_{2}}$ and $y=\mathrm{e}^{p_{2}^{\prime}-p_{2}-p_{3}}$.

A meaning of the saddle point equation (4.8) becomes clear once we regard the oriented tetrahedron in Fig. 2 as a hyperbolic ideal one; the modulus is $\mathrm{e}^{p_{2}^{\prime}-p_{2}}$, and the dihedral angles $z_{a}$ at the edges of the simplex are

$$
z_{1}=\mathrm{e}^{p_{2}^{\prime}-p_{2}}, \quad z_{2}=1-z_{1}^{-1}, \quad z_{3}=\left(1-z_{1}\right)^{-1},
$$

with opposite edges having the same angle (see e.g. Ref. 11). Then the condition (4.8) exactly coincides with the hyperbolic consistency condition for gluing three tetrahedra around central edge of RHS in Fig. 3. 
The correspondence between the saddle point condition and the hyperbolic consistency condition can be checked for other forms of the five-term relations such as $S_{2,3} S_{1,2} S_{2,3}^{-1}=$ $S_{1,2} S_{1,3}$, and a trivial identity $S_{1,2} S_{1,2}^{-1}=1$. See Ref. 7 for further correspondence between the saddle point equation and the consistency condition, which appeared in an ideal triangulation of the knot complement. As a result the oriented tetrahedron, which we have assigned to the $S$-operator based on the five-term relation (3.2), is identified with the ideal tetrahedron in the classical limit. Correspondingly the partition function of 3-manifold $M$ defined in eq. (3.7) reduces to

$$
\lim _{\gamma \rightarrow 0} 2 \mathrm{i} \gamma \log \tau(M)=\sum_{i} L\left(1-\mathrm{e}^{z_{i}}\right)
$$

which may give the hyperbolic volume and the Chern-Simons term of $M$.

We note that the function $V(x, y)$ satisfies a one parameter deformation of eq. (2.4);

$V\left(\log x, p_{1}\right)+V\left(\log \left(\frac{y}{x}\right), p_{1}+p_{2}\right)+V\left(\log \left(\frac{1-x}{1-y}\right), z\right)=V\left(\log \left(\frac{1-x^{-1}}{1-y^{-1}}\right), p_{2}\right)+V\left(\log y, p_{1}\right)$,

where $x, y$, and $z$ are given above in terms of $p_{2}, p_{3}, p_{2}^{\prime}$, and $p_{3}^{\prime}$.

\section{Physical Interpretation}

We have seen that the Faddeev integral (3.1) is closely connected with the hyperbolic geometry. Especially we have shown that classical limit of (imaginary part of) matrix elements coincides with the hyperbolic volume of the ideal tetrahedron, and that the saddle point equation coincides with the hyperbolic gluing condition. As the 3-dimensional hyperbolic geometry is nothing but the Euclidean AdS space, we recall here the so-called AdS/CFT correspondence; gravity theory on a 3-dimensional anti-de Sitter space is equivalent to the conformal field theory on the boundary. This was initiated from the observation [21] that the asymptotic symmetry group of $\mathrm{AdS}_{3}$ is generated by left and right Virasoro algebra. In this correspondence the CFT lives on the cylindrical boundary of $\mathrm{AdS}_{3}$, and in our viewpoint it seems that this cylindrical boundary can be identified with a knot $K$. 
To clarify our observation further, it is preferable to recall properties of the Faddeev integral [9, 20]. Collecting residues in integral in eq. (3.1), we get (we assume $\operatorname{Im} \gamma>0$ )

$$
\Phi_{\gamma}(\varphi)=\frac{E_{q}\left(\mathrm{e}^{\varphi}\right)}{E_{Q}\left(\mathrm{e}^{\varphi \pi / \gamma}\right)},
$$

where

$$
q=\mathrm{e}^{\mathrm{i} \gamma}, \quad Q=\mathrm{e}^{-\mathrm{i} \pi^{2} / \gamma},
$$

and $E_{q}(w)$ is the $q$-exponential function defined by

$$
E_{q}(w)=\prod_{n=0}^{\infty}\left(1+q^{2 n+1} w\right) .
$$

With this function the five-term relation (3.2) is replaced by 22

$$
E_{q}(\hat{b}) E_{q}(\hat{a})=E_{q}(\hat{a}) E_{q}\left(q^{-1} \hat{a} \hat{b}\right) E_{q}(\hat{b}),
$$

with the Weyl commuting operators

$$
\hat{a} \hat{b}=q^{2} \hat{b} \hat{a} .
$$

With a definition of a parameter $q$, and setting $\hat{a}=\exp \hat{q}$ and $\hat{b}=\exp \hat{p}$, we can see from eq. (3.3) that we can naively replace the Faddeev integral $\Phi_{\gamma}(w)$ in eq. (3.6) with $E_{q}\left(\mathrm{e}^{w}\right)$ as was shown in eq. (5.1). In fact asymptotic behavior of the $q$-exponential is same with that of $\Phi_{\gamma}(\varphi)$. We can check using the Euler-Maclaurin formula that the classical limit $\gamma \rightarrow 0$ of $E_{q}\left(\mathrm{e}^{w}\right)$ indeed reduces to the Euler dilogarithm function as in eq. (4.1) as follows;

$$
\begin{aligned}
\log E_{q}(w) & =\sum_{n=0}^{\infty} \log \left(1+\mathrm{e}^{\mathrm{i} \gamma(2 n+1)} w\right) \\
& \sim \frac{1}{2 \mathrm{i} \gamma} \int_{-w}^{0} \frac{\log (1-s)}{s} \mathrm{~d} s=\frac{1}{2 \mathrm{i} \gamma} \operatorname{Li}_{2}(-w) .
\end{aligned}
$$

Note that remaining part, $E_{Q}\left(\mathrm{e}^{\hat{\hat{p} \pi / \gamma}}\right)$, also satisfies the five-term relation (5.3) replacing $q$ by $Q$, and that the vertex operator $\mathrm{e}^{\hat{p}}$ commute with a dual vertex operator $\mathrm{e}^{\hat{p} \pi / \gamma}$. This type of modular invariance, $\gamma \leftrightarrow \pi^{2} / \gamma$, can also be seen in CFT. In this sense the integral (3.1) is a dualization of the $q$-exponential function. 
As a result, matrix element, $\left\langle p_{1}, p_{2}\left|S^{-1}\right| p_{1}^{\prime}, p_{2}^{\prime}\right\rangle$, defined in eq. (3.6) can be rewritten in terms of a function defined by

$$
Z_{\mathrm{B}}(\mu, N)=\mathrm{e}^{\frac{1}{2 \mathrm{i} \gamma}(C-\mu N)} \frac{1}{E_{q}\left(-q^{-1} \mathrm{e}^{\mu}\right)}
$$

where we set $C=\frac{\pi^{2}+\gamma^{2}}{6}+\frac{\gamma \pi}{2}$. The function $Z_{\mathrm{B}}(\mu, N)$ is familiar in CFT, and coincides with the partition function of the free boson up to constant. In this view, $\mu$ and $N$ correspond to the chemical potential and the scaled average number of angular momentum respectively. The constant term $C$ cancels if we introduce super partner whose partition function can be written as

$$
Z_{\mathrm{F}}(\mu, N)=\mathrm{e}^{\frac{1}{2 \mathrm{i} \gamma}(-C+\mu N)} E_{q}\left(-q \mathrm{e}^{\mu}\right) .
$$

This constitutes to matrix element, $\left\langle p_{1}, p_{2}|S| p_{1}^{\prime}, p_{2}^{\prime}\right\rangle$ in eq. (3.6). It should be noted that the above functions $Z_{\mathrm{B}, \mathrm{F}}(\mu, N)$ can be identified with the partition functions of bosonic (fermionic) string from the cylinder, and that the classical limit $\gamma \rightarrow 0$ corresponds to a long strip limit (Fig. 田).

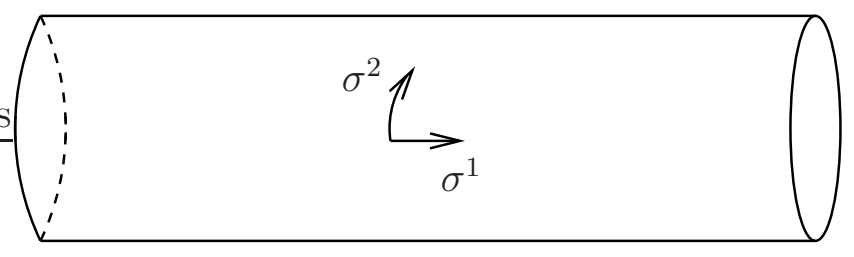

Figure 4: Cylinder in a limit $\gamma \rightarrow 0$.

It is now clear that, by replacing $\Phi_{\gamma}(\varphi)$ in eq. (3.6) with the $q$-exponential function (5.2), the bosonic string corresponds to the oriented tetrahedron (say, left in Fig. 2) while its super-partner to mirror image (say, right in Fig. 2). It seems that the quantum five-term relation (3.2) indicates a possibility to construct the quantum invariant (3.7) by using noncommutative thermodynamic variables $\mu$ and $N$ as in eq. (3.3). In this formalism, string interactions should be regarded as constraints among $\mu$ and $N$, and in the classical limit they describe the hyperbolic consistency condition.

To conclude the partition functions (5.4) - (5.5) can be seen to be fluctuating around 
the oriented ideal tetrahedron in the Euclidean $\mathrm{AdS}_{3}$. This fact seems to indicate aspects of the AdS/CFT correspondence.

\section{Concluding Remarks}

We have studied the hyperbolic structure of the partition function which is defined by use of the quantum dilogarithm function (Faddeev integral). We have shown that the classical limit of the Faddeev integral describes the ideal tetrahedron in the hyperbolic space $\mathbb{H}^{3}$ which is the space-like surface in 4-dimensional Minkowski space. Though we have originally defined the partition function in terms of the Faddeev integral, we can replace it with the $q$-exponential function as we have discussed in Section 5 . As the $q$-exponential function denotes a partition function of free bosonic and fermionic strings from cylinder, we have proposed that the hyperbolic structure $\mathbb{H}^{3}$ can be naturally embedded into (classical limit of) the CFT, and that it indicates a new aspect of the AdS/CFT correspondence. As a result, the modulus of the oriented ideal tetrahedron which is assigned to partition functions of free boson and fermion, is given explicitly in terms of the chemical potential and the average number of angular momentum, and the string interaction should be regarded as a quantization of the hyperbolic consistency conditions in gluing ideal tetrahedra. We note that we have assigned the $q$-exponential function to hyperbolic ideal tetrahedron to

construct the quantum invariant (3.7), while an idea of the Regge calculus [23] is that the three dimensional gravity is a sum over $6 j$ symbol.

Promising is a fact that the classical limit (4.10) of the partition function $\tau(M)$ is suggested to give the hyperbolic volume and the Chern-Simons invariant (2.3) of manifold $M$. As it was shown 24 that the Einstein-Hilbert action with negative cosmological constant $\Lambda=-1 / \ell^{2}$,

$$
S=\frac{1}{16 \pi G} \int \mathrm{d}^{3} x \sqrt{-g}\left(R+\frac{2}{\ell^{2}}\right)
$$

reduces to 2 sets of the Chern-Simons actions by taking a linear combination of the spin connection and the vierbein as the gauge field, the partition function $\tau(M)$ seems to a candidate for quantum gravity. 
The AdS/CFT correspondence enables us to derive the Bekenstein-Hawking entropy of black hole microscopically [25]. Therein it was shown that the entropy coincides with the asymptotic growth of the number of states in CFT with central charge $c$ [26]. We see that the computation of the central charge $c$ is essentially same with our computation of the classical limit of the partition function (4.10), once we have identified the integral $\Phi_{\gamma}(\varphi)$ with the partition function of free boson and fermion written by the $q$-exponential function. As we have seen based on the "volume conjecture" that the asymptotic behavior determines the hyperbolic geometrical structure, it would help us to understand black hole.

\section{A Dilogarithm Function}

- the Euler dilogarithm,

$$
\operatorname{Li}_{2}(z)=\sum_{n=1}^{\infty} \frac{z^{n}}{n^{2}}=-\int_{0}^{z} \mathrm{~d} s \frac{\log (1-s)}{s}
$$

- the Rogers dilogarithm,

$$
L(z)=\operatorname{Li}_{2}(z)+\frac{1}{2} \log (z) \log (1-z)
$$

- the Bloch-Wigner function,

$$
D(z)=\operatorname{Im} \operatorname{Li}_{2}(z)+\arg (1-z) \cdot \log |z|
$$




\section{References}

[1] J. M. Maldacena, Adv. Theor. Math. Phys. 2, 231 (1998).

[2] O. Aharony, S. S. Gubser, J. Maldacena, H. Ooguri, and Y. Oz, Phys. Rep. 323, 183 (2000).

[3] R. M. Kashaev, Mod. Phys. Lett. A 10, 1409 (1995).

[4] R. M. Kashaev, Lett. Math. Phys. 39, 269 (1997).

[5] H. Murakami and J. Murakami, Acta Math. 186, 85 (2001).

[6] Y. Yokota, preprint [math.QA/0009165].

[7] K. Hikami, Int. J. Mod. Phys. A (2001), to appear.

[8] S. Baseilhac and R. Benedetti, preprint [math.GT/0101234].

[9] L. D. Faddeev, Lett. Math. Phys. 34, 249 (1995).

[10] L. D. Faddeev, in Confrence Mosh Flato 1999 - Vol. I. Quantization, Deformations, and Symmetries, edited by G. Dito and D. Sternheimer, pages 149-156, Kluwer, Dordrecht, 2000.

[11] W. P. Thurston, The Geometry and Topology of Three-Manifolds, Lecture Notes in Princeton University, Princeton, 1980.

[12] J. G. Ratcliffe, Foundations of Hyperbolic Manifolds, volume 149 of Graduate Texts in Mathematics, Springer-Verlag, Berlin, 1994.

[13] D. B. A. Epstein and R. C. Penner, J. Diff. Geom. 27, 67 (1988).

[14] J. Milnor, Bull. Amer. Math. Soc. 6, 9 (1982).

[15] W. Z. Neumann and D. Zagier, Topology 24, 307 (1985).

[16] T. Yoshida, Invent. Math. 81, 473 (1985). 
[17] W. D. Neumann, in Topology '90, pages 243-271, de Gruyter, Berlin, 1992.

[18] W. D. Neumann and J. Yang, Duke Math. J. 96, 29 (1999).

[19] L. Chekhov and V. V. Fock, Theor. Math. Phys. 120, 1245 (1999).

[20] L. D. Faddeev, R. M. Kashaev, and A. Y. Volkov, Commun. Math. Phys. 219, 199 (2001).

[21] J. D. Brown and M. Henneaux, Commun. Math. Phys. 104, 207 (1986).

[22] L. D. Faddeev and R. M. Kashaev, Mod. Phys. Lett. A 9, 427 (1994).

[23] G. Ponzano and T. Regge, in Spectroscopic and Group Theoretical Methods in Physics, edited by F. Bloch, pages 1-58, North-Holland, 1968.

[24] E. Witten, Nucl. Phys. B 311, 46 (1988/1989).

[25] A. Strominger and C. Vafa, Phys. Lett. B 379, 99 (1996).

[26] J. L. Cardy, Nucl. Phys. B 270, 186 (1986). 